Pacific Journal of Mathematics

AN IMBEDDING SPACE FOR SCHWARTZ DISTRIBUTIONS 


\title{
AN IMBEDDING SPACE FOR SCHWARTZ DISTRIBUTIONS
}

\author{
Donald E. MYers
}

1. Introduction. We consider here a facet of the problem of justifying the methods of the operational calculus and in particular the use of the "Dirac Delta Function". L. Schwartz's “Theorie des Distributions" [6] is the most complete exposition to date on generalized functions but the operational calculus as such is largely omitted. B. Van Der Pol [8] discusses the latter but not in the context of distributions. Ketchum and Aboudi [4] suggested using unilateral Laplace Transforms to construct a link between Schwartz's theory and the operational calculus. This paper will enlarge on the latter suggestion. Two principal results are obtained. An imbedding space is constructed and a comparison between the topologies is made.

Let $S$ denote the strip $\sigma_{1}<R(z)<\sigma_{2}$, in the complex plane. Consider the one parameter family of functions $\left\{e^{z t}\right\}$, where the parameter $z$ ranges over $S$ and $-\infty<t<\infty$. This family is not a linear space but each member possesses derivatives of all orders. In a manner analogous to Schwartz we define an $\mathrm{L}_{s}$-Distribution to be an analytic complexvalued functional on the above family of functions, where by analytic we mean with respect to the parameter $z$. If $\alpha$ is any complex scalar and $F, \sigma$ are two such functionals then we require that $F \cdot e^{z t}+\sigma \cdot e^{z t}-$ $(F+\sigma) \cdot \sigma^{z t}$, and $(\alpha F) \cdot e^{z t}=F \cdot\left(\alpha e^{z t}\right)$. The latter property then allows us to define the derivative in a manner similar to that of Schwartz, that is $F^{\prime} \cdot e^{z t}=F \cdot\left(e^{z t}\right)^{\prime}=F \cdot z e^{z t}=z F \cdot e^{z t}$. It also follows that the Laplace Transform supplies an integral representation of some of the functionals. The other $\mathrm{L}_{S}$-Distributions define generalized functions for similar integral representations. That is, each function analytic for $z \in S$ has for its values, the values of an $\mathrm{L}_{S}$-Distribution acting on a function $e^{z t}$ and the $\mathrm{L}_{\mathrm{\varsigma}}$-Distribution has an integral representation utilizing the symbolic inverse Laplace Transform of the analytic function. In most of this paper we deal only with analytic functions whose inverse trans forms exist but the definitions and theorems will be stated without this restriction where possible. Following a practice used by other authors, we will call the inverse Laplace Transform, symbolic or not, an $\mathbf{L}_{S^{-}}$ Distribution rather than the functional. Because of the relation between the functional and an analytic function we concentrate on the latter and utilize the already known properties of such functions. By emphasizing the integral representations rather than the functionals we utilize the

Received January 3, 1961. Based on the author's thesis at the University of Illinois, 1960 (unpublished). 
Riesz Representation Theorem for continuous linear functionals to establish a correspondence to Schwartz Distributions.

As stated above each functional has a representation by an analytic functions, using this we will define convergence in a fashion similar to that of Schwartz. That is, a sequence of $\mathrm{L}_{S}$-Distributions will converge if the sequence of values, when operating on an arbitrary member of the one-parameter, converges. Because of the parameterization this definition can be stated directly in terms of the representations by the analytic functions.

\section{2. $\mathrm{L}_{S}-$ Distributions.}

DEFINITION 1. If an $\mathrm{L}_{S}$-Distribution is determined by an analytic function $f(z)$, then $f(z)$ is its bilateral Laplace Transform. Denote this $\mathrm{L}_{S}$-Distribution by $[f(z)]_{t}$ or $f_{t}$. Further, abbreviate $L_{S}$-Distribution by $\mathrm{L}_{s}-D$.

Definition 2. The derivative of an $\mathrm{L}_{S}-\mathrm{D},[f(z)]_{t}=f_{t}$ is the $\mathrm{L}_{S}-\mathrm{D}$, $[z f(z)]_{t}=f_{t}^{\prime}$. For a fixed $S$, the set of all $\mathrm{L}_{s}-\mathrm{D}$ 's is metrized by a Frechet type metric on the transforms. See [7], page 137. For a pair of functions $f(z), g(z)$ analytic in $S$, denote the metric by $N_{s}(f, g)$. The following property of this metric could have been used a definition since it is the only property used in this paper.

THEOREM 3. A sequence of functions, all analytic in $S$, converges with respect to the metric $N_{S}$ if and only if the sequence converges uniformly on every compact subset of $S$.

Definition 4. $\rho_{S}\left(f_{t}, g_{t}\right)=N_{S}(f, g)$ where $f_{t}, g_{t}$ are the $\mathrm{L}_{S}$-D's whose transforms are $f(z), g(z)$ respectively.

DEFINITION 5. If $f(z)$, analytic in $S$, is the bilateral Laplace Transform of a point-function $F(t)$, then $F(t)$ is called a Point-Function $\mathrm{L}_{s}-\mathrm{D}$ or P.F.L $L_{S}-\mathrm{D}$.

Theorem 6. If $F_{i}(t), i=0,1,2,3, \cdots$ all possess bilateral Laplacə Transforms analytic in a strip $S, \sigma_{1}<R(z)<\sigma_{2}$, and

$$
\begin{aligned}
& \int_{0}^{\infty}\left|e^{-\sigma_{1} t} F_{i}(t)\right|^{2} d t<\infty, \\
& \int_{-\infty}^{0}\left|e^{-\sigma_{2} t} F_{i}(t)\right|^{2} d t<\infty
\end{aligned}
$$

for all $i$, then let 


$$
\begin{aligned}
d\left(F_{k}, F_{j}\right)= & {\left[\int_{0}^{\infty} e^{-2 \sigma_{1} t}\left|F_{k}(t)-F_{j}(t)\right|^{2} d t\right]^{1 / 2} } \\
& +\left[\int_{-\infty}^{0} e^{-2 \sigma_{2} t}\left|F_{k}(t)-F_{j}(t)\right|^{2} d t\right]^{1 / 2} .
\end{aligned}
$$

If $d\left(F_{i}, F_{0}\right) \rightarrow 0$ as $i \rightarrow \infty$ then

$$
F_{i}(t) \rightarrow F_{0}(t) \text { as P.F.L } S_{S} \text { D's. }
$$

Proof. Write the transform of $F_{i}(t)-F_{0}(t)$ as

$$
\begin{aligned}
& \int_{-\infty}^{0} e^{-t\left(z-\sigma_{2}\right)} e^{-\sigma_{2} t}\left[F_{i}(t)-F_{0}(t)\right] d t \\
& \quad+\int_{0}^{\infty} e^{-t\left(z-\sigma_{1}\right)} e^{-\sigma_{1} t}\left[F_{i}(t)-F_{0}(t)\right] d t .
\end{aligned}
$$

By the Cauchy-Schwartz Inequality

$$
\begin{aligned}
& \left|f_{i}(z)-f_{0}(z)\right| \\
& \leqq \\
& \quad\left[\frac{1}{2\left[\sigma_{2}-R(z)\right]} \int_{-\infty}^{0} e^{-2 \sigma_{2} t}\left|F_{i}(t)-F_{0}(t)\right|^{2} d t\right]^{1 / 2} \\
& \quad+\left[\frac{1}{2\left[R(z)-\sigma_{1}\right]} \int_{\sigma}^{\infty} e^{-2 \sigma_{1} t}\left|F_{i}(t)-F_{0}(t)\right|^{2} d t\right]^{1 / 2} .
\end{aligned}
$$

If

$$
g(z)=\max _{z \in S}\left[\sqrt{\frac{1}{2\left(R(z)-\sigma_{1}\right)}}, \sqrt{\frac{1}{2\left(\sigma_{2}-R(z)\right)}}\right]
$$

then

$$
\left|f_{i}(z)\right| \leqq g(z) g\left(F_{i}, F_{0}\right)
$$

and hence $f_{2}(z) \rightarrow f_{0}(z)$ uniformly on each compact subset in $S$ if $d\left(F_{i}, F_{0}\right) \rightarrow$ 0 as $i \rightarrow \infty$.

An interpretation of Theorem 6 might be that if $\left\{e^{-\sigma_{1} t} F_{i}(t)\right\}$ converges in $L_{2}[0, \infty]$ to $e^{-\sigma_{1} t} F_{0}(t)$ and $\left\{e^{-\sigma_{3} t} F_{i}(t)\right\}$ converges in $L_{2}[\infty, 0]$ to $e^{-\sigma_{2} t} F_{0}(t)$ and each $F_{i}(t)$ has a bilateral Laplace Transform then the sequence of P.F.L $L_{S}-\mathrm{D}$ 's converges with respect to the metric $\rho_{S}$.

THEOREM 7. Let $f_{j}(z), j=0,1,2,3, \cdots$ be an infinite sequence of functions analytic in a strip $S, \sigma_{1}<R(z)<\sigma_{2}$, and further suppose there exists a $C$ such that $\left|f_{j}(z)\right|<C e^{-\eta_{0}|I(z)|}$ for some $\eta_{0}>0$, in all of S. If $N_{s}\left(f_{j}, f_{0}\right) \rightarrow 0$ as $j \rightarrow \infty$ then $F_{j}(t) \rightarrow F_{0}(t)$ uniformly on every bounded interval in the t-line. $F_{j}(t)$ denotes the inverse bilateral Laplace Transform of $f_{j}(z)$.

Proof. The hypothesis is sufficient to ensure the existence of the 
inverse transform of each $f_{j}(z),[2]$. That is,

$$
\begin{aligned}
F_{j}(t)= & \int_{-\infty}^{\infty} \frac{e^{x t}}{2 \pi}\left[e^{i t y} f_{j}(x+i y)\right] d y \\
& \text { for } \sigma_{1}<x<\sigma_{2} .
\end{aligned}
$$

Then

$$
\begin{aligned}
& \left|F_{j}(t)-F_{0}(t)\right| \leqq \frac{e^{x t}}{2 \pi}\left|\int_{-\infty}^{-\rho} e^{i t y}\left[f_{j}(x+i y)-f_{0}(x+i y)\right] d y\right| \\
& +\frac{e^{x t}}{2 \pi}\left|\int_{-\rho}^{p} e^{i t y}\left[f_{j}(x+i y)-f_{0}(x+i y)\right] d y\right| \\
& \quad+\frac{e^{x t}}{2 \pi}\left|\int_{\rho}^{\infty} e^{i t y}\left[f(x+i y)-f_{0}(x+i y)\right] d y\right| .
\end{aligned}
$$

For $\varepsilon>0$, and $a<t<b$, let $\rho$ be such that $\frac{2 e^{\sigma_{2} b} C^{\prime} e^{-\eta_{0} \rho}}{{ }^{\pi} \eta_{0}}<\varepsilon / 2$ and $J$ such and $J$ such that $\left[\frac{e^{\sigma_{2} b}}{\pi}\right] N_{S}\left(f_{j}, f_{0}\right)<\varepsilon / 2$ for $j>J$, then

$$
\begin{gathered}
\left|F_{j}(t)-F_{0}(t)\right|<\varepsilon \text { for } j>\text { and } \\
a \leqq t \leqq b
\end{gathered}
$$

TheOREM 7.1. If in Theorem $7, \sigma_{1}<0<\sigma_{2}$ then $F_{j}(t) \rightarrow F_{0}(t)$ uniformly for $-\infty<t<\infty$.

Definition 8. For each $\mathrm{L}_{s}-\mathrm{D}, f_{t}$, define $f_{t+n}$ to be $\left[e^{h z} f(z)\right]_{t}$.

THEOREM 9. If $f_{t}$ is an arbitrary $\mathrm{L}_{s}-\mathrm{D}$ then

$$
\rho\left(\frac{f_{t+h}-f_{t}}{h}, f_{t}^{\prime}\right) \rightarrow 0 \text { as } h \rightarrow 0 .
$$

Proof. By definition

$$
\begin{aligned}
& f_{t}^{\prime}=[z f(z)]_{t} \\
& f_{t+h}=\left[e^{h z} f(z)\right]_{t}
\end{aligned}
$$

so that

$$
\begin{aligned}
& \frac{f_{t+h}-f_{t}}{h}-f_{t}^{\prime} \\
& =\left[\frac{e^{h z} f(z)-f(z)-z f(z)}{h}\right]_{t} \\
& =\left[f(z)\left[\frac{h z^{2}}{2 !}+\frac{h^{2} z^{3}}{3 !}+\cdots\right]\right]_{t}
\end{aligned}
$$


and since

$$
\frac{h z^{2}}{2 !}+\frac{h^{2} z^{3}}{3 !}+\cdots \rightarrow 0
$$

uniformly on each compact set in $S$ as $h \rightarrow 0$, the theorem is proved.

DEFinition 10. An $\mathrm{L}_{S}$-D $f_{t}$ is said to have point-values $F(t)$ for $c<t<d$ if there exists a $\sigma(t)$ such that for some $k, f(z)=z^{k} g(z), g(z)$ being the bilateral Laplace Transform of $\sigma(t)$ and finally that $\sigma^{(k)}(t)=$ $F(t)$ for $(c<t<d)$.

For example $[1]_{t}$ has zero-point values in every open interval, in the $t$-line, that does not contain the point $t=0$. Since

$$
H(t)=\left\{\begin{array}{l}
1, t>0 \text { has for } \\
0, t>0
\end{array}\right.
$$

its transform $1 / z$ and $[1]_{t}=\left[z \frac{1}{z}\right]_{t}$ finally $H^{\prime}(t)=0$ for all $t \neq 0 . \quad[1]_{t}$ is the "Dirac Delta Function".

THEOREM 11. If $\left\{_{n} f_{t}\right\}$ is a sequence of $\mathrm{L}_{s}$-D's converging to an $\mathrm{L}_{S}-\mathrm{D}$ ${ }_{0} f_{t}$ then $\left\{{ }_{n} f_{t}^{(k)}\right\}$ converges to ${ }_{0} f_{t}^{(k)}$ for all $k=0,1,2, \ldots$

Proof. By definition $\left\{{ }_{n} f_{t}\right\}$ converges to

$$
{ }_{0} f_{t} \leftrightarrows \max _{z \in K}\left|{ }_{n} f(z)-{ }_{0} f(z)\right| \rightarrow 0
$$

as $n \rightarrow \infty$ for all compact $K \subset S$. Since in the complex plane, a set is compact $\leftrightarrows$ if it is closed and bounded, there exists an $M_{K}$ for each $K|z| \leqq M_{K}$ for $z \in k$.

Then $\max _{z \in K}\left|{ }_{n} f(z)-{ }_{0} f(z)\right| \rightarrow 0 \leftrightarrows$

$$
\left|M_{K}\right|{ }^{k}{ }_{n} f(z)-f(z) \mid \rightarrow 0 \text { as } n \rightarrow \infty \text { for each fixed }
$$

positive integer $k$ apply Definition 2 .

ExAmple. The following will be used as a counter-example in the last section. Consider the Taylor-expansion for

$$
e^{-z}=1-z+\frac{z^{2}}{2 !}-\frac{z^{3}}{3 !}+\cdots+\frac{(-1)^{n} z^{n}}{n !}+\cdots
$$

$\left[e^{-z}\right]_{t}$ is the "Delta Dirac Function" translated so that $\mathrm{L}_{S}-\mathrm{D}$ has zero point-values for all $t$ except for

$$
t=1 . \quad\left[(-1)^{n} \frac{z^{n}}{n !}\right]_{t}=\frac{(-1)^{n}}{n !}\left[z^{n}\right]_{t}=\frac{(-1)}{n !}[1]_{t}^{(n)} .
$$


The latter $\mathrm{L}_{S}-\mathrm{D}$ has zero point-values for all $t \neq 0$. Since the Taylor Series converges for all $z$ and hence uniformly for compact sets the series of $\mathrm{L}_{S}-\mathrm{D}$ 's converges

3. General $\mathrm{L}_{S}$-Distribution. The set of all $\mathrm{L}_{S}-\mathrm{D}$ 's for any fixed $S$ does not contain a subset isomorphic with the set point-functions having pointvalues a.e. For example, the function $F(t)=1$ does not have a transform even though it is continuous for $-\infty<t<\infty$. However each member of the sequence of functions

$$
\begin{aligned}
F_{i}(t) & =1, & & (-i \leqq t \leqq i) \\
& =0, & & (t>i, t<-i) \\
i & =0, & & (0,1,2,3,4, \cdots)
\end{aligned}
$$

does possess a transform. Further for each open interval $(c, d)$ only a finite number of the elements of the sequence have different point-values than $F(t)$ in $(\mathrm{c}, d)$. The sequence represents $F(t)$.

Definition 12. A sequence $\left\{_{n} f_{t}\right\}$ of $\mathrm{L}_{S}$-D's is called Fundamental if for each open interval $(c, d)$ there exists an integer $N$ such that for $n>N_{n} f_{t}-{ }_{n+p} f_{t}, p=0,1,2,3, \cdots$ is an $\mathrm{L}_{S}$ - $\mathrm{D}$ with zero point-values in $(c, d)$. Fundamental sequence of $\mathrm{L}_{s}-\mathrm{D}$ in abbreviated by F.S.S.

Definition 13. Two F.S.S.'s, $\left\{{ }_{n} g_{t}\right\}$ and $\left\{_{n} f_{t}\right\}$ are said to be Similar if for each open interval $(c, d)$ there exists an integer $N$ such that for $n>N_{n} g_{t}-{ }_{n} f_{t}$ is an $\mathrm{L}_{s}-\mathrm{D}$ with zero pointvalues in $(c, d)$.

Lemma 14. The Similarity defined in Definition 13 for pairs of F.S.S.'s is an Equivalence relation and is invariant under addition and differentiation.

THEOREM 15. The equivalence classes under the Similarity relation are called G. $\mathrm{L}_{S}-\mathrm{D}$ 's or General $\mathrm{L}_{s}-\mathrm{D}$ 's. They form an Abelian group, closed with respect to scalar multiplication and differentiation.

The Representation Theorem.

Theorem 16. Let $A$ denote the entire complex plane, then there is a subset, $D$, of the set of all $G$. $\mathrm{L}_{A}$-D's that is isomorphic with the set of all Schwartz Distributions. The isomorphism is invariant with respect to addition, scalar multiplication and differentiation.

(a) By definition, a Schwartz Distribution is a linear functional on the space of infinity differentiable point-functions with compact supports and is continuous when restricted to the set. Each Schwartz Distribution has an integral respresentation when restricted to a bounded closed 
interval, [3]. This representation has the form

$$
D_{t}(\phi)=(-1)^{r} \int_{a}^{b} F(t) \phi^{(r)} d t
$$

where $F(t)$ is continuous on $[a, b]$ and $r$ is an integer dependent on $[a, b]$ and the distribution $D_{t} . \phi(t)$ is any function with support the closed interval $[a, b]$. Let $\left[a_{n}, b_{n}\right]$ be a sequence of intervals where $-\infty \leftarrow$ $a_{n+1} \leqq a_{n} \leqq b_{n} \leqq b_{n+1} \rightarrow \infty$ as $n \rightarrow \infty$. For each $n$ there is an $F_{n}(t)$ and an $r_{n}$. Let

$$
\begin{aligned}
G_{n}(t) & =(-1)^{r} F_{n}(t), & & \left(a_{n} \leqq t \leqq b_{n}\right) \\
& =0, & & \left(t>b_{n}, t<a_{n}\right) .
\end{aligned}
$$

Then let

$$
f_{n}(z)=\int_{a_{n}}^{b_{n}} e^{-z t} z^{r_{n}} G_{n}(t) d t .
$$

It remains to be shown that the sequence $\left\{_{n} f_{t}\right\}$ is an F.S.S. and that the equivalence class is independent of the sequence of covering intervals. The G. $L_{S}-\mathrm{D}$ determined is the representative of $D_{t}$.

(b) Let $I$ be an arbitrary open interval in the $t$-line, denoted $(c, d)$. There exists an $N$ then such that for $n>N\left[a_{n}, b_{n}\right] \supset(c, d)$. Let $\mathbb{F}_{n}(t)$, $F_{n+p}(t), r_{n}, r_{n+p}$ be the continuous functions and integers given for the representation of the distribution $D_{t}$ on the intervals $\left[a_{n}, b_{n}\right]$ and $\left[a_{n+p}\right.$, $\left.b_{n+p}\right]$ respectively. Using Halperin's notation, let $S\left[a_{n}, b_{n}\right]$ denote the class of testing functions associated with the interval $\left[a_{n}, b_{n}\right]$ that is, if $\phi \in S\left[a_{n}, b_{n}\right]$ then $\phi^{(k)}(t)$ is zero for $t \notin\left[a_{n}, b_{n}\right]$, and $\phi^{(k)}(t)$ exists for all $t \in\left[a_{n}, b_{n}\right]$ for $k=0,1,2,3, \cdots$. It is seen that $S\left[a_{n}, b_{n}\right] \subset S\left[a_{n+p}, b_{n+p}\right]$. If $\phi \in S\left[a_{n}, b_{n}\right] \subset S\left[a_{n \vdash p}, b_{n \vdash p}\right]$ then

$$
\begin{aligned}
D_{t}(\phi) & =(-1)^{r_{n}} \int_{a_{n}}^{b_{n}} F_{n}(t) \phi^{\left(r_{n}\right)}(t) d t \\
& =(-1)^{r_{n+p}} \int_{a_{n+p}}^{b_{n+p}} F_{n+p}(t) \phi^{\left(r_{n+p}\right)}(t) d t
\end{aligned}
$$

or

$$
\int_{a_{n}}^{b_{n}}\left[F_{n}(t) \phi^{\left(r_{n}\right)}(t)-(-1)^{r_{n+p}-r_{n}} F_{n+p}(t) \phi^{\left(r_{n+p}\right)}(t)\right] d t=0
$$

since

$$
\phi^{(k)}(t)=0 \text { for } t \notin\left[a_{n}, b_{n}\right]
$$

Let

$$
T \cdot F_{n}(t)=\int_{a_{n}}^{t} F_{n}(x) d x
$$




$$
T^{2} \cdot F_{n}(t)=T \cdot\left[T \cdot F_{n}(t)\right]
$$

Then if $r_{n+p} \geqq r_{n}$

$$
0=\int_{a_{n}}^{b_{n}}\left[(-1)^{r_{n+p-r_{n}}} T^{r_{n+p-r_{n}}} \cdot F_{n}(t) \phi(t)^{\left(r_{n+p}\right)}-F_{n+p}(t) \phi^{\left(r_{n+p}\right)}(t)\right] d t .
$$

It follows then that

$$
(-1)^{r_{n+p}-r_{n}} T^{r_{n+p-r_{n}}} \cdot F_{n}(t)-F_{n+p}(t)
$$

is a polynomial $Q_{m}(t)$ of degree $m \leqq r_{n+p}-1$ for $a_{n} \leqq t \leqq b_{n}$. Similar results are obtained if $r_{n} \geqq r_{n}+p$.

$$
\begin{aligned}
{ }_{n+p} f(z)-{ }_{n} f(z)= & \int_{a_{n+p}}^{b_{n+p}} e^{-z t} z^{r_{n+p}} G_{n+p}(t) d t \\
& -\int_{a_{n}}^{b_{n}} e^{-z t} z^{r_{n}} G_{n}(t) d t .
\end{aligned}
$$

Using $Q_{m}(t)$ we have

$$
\begin{aligned}
{ }_{n+p} f(z)-{ }_{n} f(z)= & \int_{a_{n+p}}^{a_{n}} e^{-z t} z^{r_{n+p}} G_{n+p}(t) d t \\
& +\int_{a_{n}}^{b_{n}} e^{-z t} z^{r_{n} \uparrow p} Q_{m}(t) d t \\
& +\int_{b_{n}}^{b_{n+p}} e^{-z t} z^{r_{n+p}} G_{n+p}(t) d t .
\end{aligned}
$$

The first integral can be considered as the transform of the $r_{n+p}$ th derivative of a function with zero point-values exterior to the interval $\left[a_{n+p}, a_{n}\right]$ and hence interior to the interval $(c, d)$. The second integral can be considered as the transform of the $r_{n+p}$ th derivative of a polynormial of degree less than or equal to $r_{n+p}-1$. Hence the $\mathrm{L}_{S}-\mathrm{D}$ determined has zero pointvalues on the interior and exterior of the interval $\left[a_{n}, b_{n}\right]$ and hence on the interior of $(c, d)$. This $\mathrm{L}_{S}$-D may not have zero point-values at $t=a_{n}$ or $t=b_{n}$. Finally then the third integral considered as a transform determines an $L_{S}-D$ with zero pointvalues exterior to the interval $\left[b_{n}, b_{n+p}\right]$ and hence on the interior of $(c, d)$. ${ }_{n+p} f_{t}-{ }_{n} f_{t}$ is an $\mathrm{L}_{s}-\mathrm{D}$ with zero pointvalues on the interior of $(c, d)$, if $n>N$. The sequence constructed in part (a) is an F.S.S.

(c) Suppose $\left[a_{n}, b_{n}\right]$ and $\left[c_{n}, d_{n}\right]$ are two expanding sequence of closed intervals covering the real line. Let $\left\{_{n} f_{t}\right\}$ and $\left.{ }_{n} g_{t}\right\}$ be the F.S.S.'s obtained from the consturuction of part (a) using the former sequences. Let $I$ be an arbitrary open interval in the $t$-line. Then there exists integers $N_{I}^{1}, N_{I}^{2}$ such ${ }_{n+p} f_{f}-{ }_{n} f_{t}$ for $n<N_{I}^{1}$ and ${ }_{n+p} g_{t}-{ }_{n} g_{t}$ for $n \leqq N_{I}^{2}$ $\mathrm{L}_{S}$-D's with zero point-values for $t \in I$. Further there exists an integer $M \ni\left[a_{n}, b_{n}\right] \subset\left[C_{N}, d_{N}\right]$ for $n \geqq M$ and $N=N_{l}^{2}$. Consider 


$$
{ }_{n} f_{\mathcal{J}}-{ }_{n} g_{t}={ }_{n} f_{t}-K f_{t}+K f_{t}-K g_{t}+K g_{t}-{ }_{n} g_{t}
$$

where $K$ is the largest of $N_{I}^{1}, N_{I}^{2}, M$. For $n>K$ then the first difference on the right is an $\mathrm{L}_{S}-\mathrm{D}$ with zero point-values for $t \in I$ since $\left\{f_{t}\right\}$ is an. F.S.A. The second difference can be shown to be an $\mathrm{L}_{S}-\mathrm{D}$ with zero point-values for $t \in I$ by the method of part (b). Finally the third difference is an $\mathrm{L}_{S}-\mathrm{D}$ with zero point-values for $t \in I$ since $\left.{ }_{n} g_{t}\right\}$ is F.S.A. The two F.S.A.'s are similar and hence determine the same G. $\mathrm{L}_{A} . \mathrm{D}$. The correspondence between the Schwartz Distribution and the G. $\mathrm{L}_{4} \cdot-\mathrm{D}$. is one-to-one. The invariance of this isomorphism with respect to addition, differentiation and scalar multiplication follows from Lemma 14.

\section{A Topology for G.L-D's}

Definition 16. An F.S.S. $\left\{{ }_{n} f_{t}\right\}$ is said to have point-values $F(t)$ for $t \in(c, d)$, an open interval, if there exist an integer $N_{(c, a)}$ such that for $n>N,{ }_{n} f_{t}$ is an $\mathrm{L}_{s}-\mathrm{D}$ possessing pointvalues $F(t)$ for $t \in(c, d)$. A G. $\mathrm{L}_{s}-\mathrm{D}$ is said to have pointvalues $F(t)$ for $t \in(c, d)$ if there is an F.S.S. unit equivalence class possessing that property.

DEFinition 17. Let $\left\{_{n} f_{t}\right\}_{1}, \cdots,\left\{_{n} f_{t}\right\}_{i}, \cdots$ be a sequence of F.S.S.'s. Denote the $n$th element of the $j$ th F.S.S. by $\left({ }_{n} f_{t}\right)_{j}$. Then sequence is said to converge to the sequence of $\mathrm{L}_{s}-\mathrm{D}$ 's $\left.{ }_{n} f_{t}\right\}_{0}$, if for $\varepsilon>0$ there exist integers $N_{\varepsilon}, J_{\varepsilon}$ such that $\left.\rho\left[{ }_{n} f_{t}\right)_{j},\left({ }_{n} f_{t}\right)_{0}\right]<\varepsilon$ when $n>N_{\varepsilon}, j>J_{\varepsilon}$.

Definition 18. Let $D_{1}, D_{2}, \cdots$ be a sequence of G.L $\mathrm{L}_{s}$-D's. Further suppose $L_{1}, L_{2}, \cdots$ is a sequence of F.S.S.'s each having support [a,b] and that for each $j=1,2, \cdots L_{j}$ represents $D_{j}$ is $(a, b)$. That is, for some F.S.S. in $D_{j}$, the difference of $L_{j}$ and the F.S.S. has zero pointvalues in $(a, b)$. Then if $L_{1}, L_{2}, \cdots$ is convergent in the sense of Definition $17, D_{1}, D_{2}, \cdots$ is said to converge to $D_{0}$ where $D_{0}$ is the G.L $\mathrm{L}_{S}-\mathrm{D}$. determined by $L_{0}$.

THEOREM 19. If a sequence of Schwartz Distributions is convergent in an open interval $(a, b)$ in Schwartz's sense then the sequence of $\mathrm{G}_{\mathrm{H}} \mathrm{L}_{s}-\mathrm{D}$ 's isomorphic to the respective Schwartz Distributions is convergent in the interior of every closed interval contained in $(a, b)$.

Proof. Let $D_{1}, D_{2}, \cdots$ be a sequence of Schwartz Distributions convergent in Schwartz's sense in $(a, b)$. For any closed interval $[c, d]$ contained in $(a, b)$ there exists a sequence of representation

$$
D_{i}(\phi)=(-1)^{r_{c}} \int_{c}^{a} F_{i}(t) \phi(t)^{\left(r_{c}\right)} d t
$$


for $\phi \in S[c, d]$. Since the sequence $D_{1}, D_{2}, \ldots$ is convergent there exists one integer $r_{c}$ which can be used in all the representations and also the limit representation.

For each $j$, construct the F.S.A. $\left\{{ }_{n} f_{t}\right\}_{j}$ where $\left\{_{n} f_{t}\right\}_{j}=\left(f_{t}\right)_{j}$ and

$$
(f)_{j}=\left[\int_{c}^{a} e^{-z t} G_{j}(t)_{z}{ }^{r_{c}} d t\right]_{t}
$$

where

$$
\begin{aligned}
G_{j}(t) & =(-1)^{r_{c}} F_{\jmath}(t), & & c \leqq t \leqq d \\
& =0, & & \text { otherwise . }
\end{aligned}
$$

Since the sequence of Distributions is convergent

$$
\begin{aligned}
\lim T^{r_{c}} F_{j}(t) & \left.=F_{0}(t) \text { [uniformly }[c, d]\right] j \rightarrow \infty \\
K= & \left|\int_{c}^{a} z^{r_{c}} e^{-z t}\left[T^{r_{c}} G_{j}(t)-G_{0}(t)\right] d t\right| \\
& \leqq|d-c| e^{-c \sigma_{1}}\left|T^{r_{c}} G_{j}(t)-G_{a}(t)\right|\left|d^{r_{0}}\right| .
\end{aligned}
$$

It follows that

$$
\begin{aligned}
K & =\left|(f(z))_{j}-(f(z))_{0}\right| \leqq M \varepsilon \\
\text { for } j>J_{\varepsilon}, M & =|d-c| e^{-c \sigma_{1}}\left|\sigma_{2}^{r_{c}}\right| .
\end{aligned}
$$

Then $(f(z))_{j} \rightarrow(f(z))_{0}$ uniformly on every compact set in the strip $\sigma_{1} \leqq$ $R(z) \leqq \sigma_{2}$ and hence in the metric $\rho_{s}$. By definition then $\left(f_{t}\right)_{j} \rightarrow\left(f_{t}\right)_{0}$ and hence

$\left\{f_{t}\right\}_{1}, \cdots,\left\{_{n} f_{t}\right\}_{j}, \cdots$ converges to $\left\{_{n} f_{t}\right\}_{0}$ in the interior of $(c, d)$. The sequence of $\mathrm{G}$. $\mathrm{L}_{s}$-D's converges for $t \in(c, d)$.

The example given earlier for a series representation of "Delta" Distribution with a discontinuous at $t=1$ converges in the sense defined herein but not in Schwartz's sense. The $\mathrm{L}_{s}-\mathrm{D}\left[e^{-z}\right]_{t}$ and its series representation furnish a solution to the differential equation

$$
f(x-1)=f(x)+f^{\prime}(x)+\frac{f^{\prime \prime}(x)}{2 !}+\cdots+\frac{f^{(n)}(x)}{n !}+\cdots
$$

\section{BIBLIOGRAPHY}

1. S. Bochner, Lectures on Fourier Integrals, Princeton University Press, Princeton, 1956.

2. G. Doetsch, Theorie und Andwending der Laplace Transformation, Juluis Springer, Berlin, 1937.

3. I. Halperin, Introduction to the Theory of Distributions, University of Toronto Press, Toronto, Canada, 1952.

4. P. Ketchum, and R. Abondi, Schwartz Distributions, Proceedings of the Second Midwest Symposium on Circuit Theory, Michigan State University, 1956.

5. D. Myers, An Imbedding Space for Schwartz Distributions, Thesis University of Illinois, 
1960.

6. L. Schwartz, Theorie des Distributions, I et II, Herman et Cie, Paris, 1950-1951.

7. W. Thron, Introduction to the Theory of Functions of a Complex Variable, John Wiley, New York, 1953.

8. B. Van Der Pol, Operational Calculus, Cambridge University Press, 1950. 



\section{PACIFIC JOURNAL OF MATHEMATICS}

\section{EDITORS}

\author{
RaLPh S. Phillips \\ Stanford University \\ Stanford, California \\ F. H. BRowNELL \\ University of Washington \\ Seattle 5 , Washington
}

A. L. Whiteman

University of Southern California

Los Angeles 7, California

L. J. Paige

University of California

Los Angeles 24, California

\author{
E. F. BECKENBACH \\ T. M. CHERRY
}

\author{
ASSOCIATE EDITORS

$\begin{array}{lll}\text { D. DERRY } & \text { H. L. ROYDEN } & \text { E. G. STRAUS } \\ \text { M. OHTSUKA } & \text { E. SPANIER } & \text { F. WOLF }\end{array}$

\section{SUPPORTING INSTITUTIONS}

\author{
UNIVERSITY OF BRITISH COLUMBIA \\ CALIFORNIA INSTITUTE OF TECHNOLOGY \\ UNIVERSITY OF CALIFORNIA \\ MONTANA STATE UNIVERSITY \\ UNIVERSITY OF NEVADA \\ NEW MEXICO STATE UNIVERSITY \\ OREGON STATE COLLEGE \\ UNIVERSITY OF OREGON \\ OSAKA UNIVERSITY \\ UNIVERSITY OF SOUTHERN CALIFORNIA
}

\author{
STANFORD UNIVERSITY \\ UNIVERSITY OF TOKYO \\ UNIVERSITY OF UTAH \\ WASHINGTON STATE COLLEGE \\ UNIVERSITY OF WASHINGTON \\ AMERICAN MATHEMATICAL SOCIETY \\ CALIFORNIA RESEARCH CORPORATION \\ HUGHES AIRCRAFT COMPANY \\ SPACE TECHNOLOGY LABORATORIES \\ NAVAL ORDNANCE TEST STATION
}

Mathematical papers intended for publication in the Pacific Journal of Mathematics should be typewritten (double spaced), and the author should keep a complete copy. Manuscripts may be sent to any one of the four editors. All other communications to the editors should be addressed to the managing editor, L. J. Paige at the University of California, Los Angeles 24, California.

50 reprints per author of each article are furnished free of charge; additional copies may be obtained at cost in multiples of 50 .

The Pacific Journal of Mathematics is published quarterly, in March, June, September, and December. The price per volume (4 numbers) is $\$ 12.00$; single issues, $\$ 3.50$. Back numbers are available. Special price to individual faculty members of supporting institutions and to individual members of the American Mathematical Society: $\$ 4.00$ per volume; single issues, $\$ 1.25$.

Subscriptions, orders for back numbers, and changes of address should be sent to Pacific Journal of Mathematics, 103 Highland Boulevard, Berkeley 8, California.

Printed at Kokusai Bunken Insatsusha (International Academic Printing Co., Ltd.), No. 6, 2-chome, Fujimi-cho, Chiyoda-ku, Tokyo, Japan.

\section{PUBLISHED BY PACIFIC JOURNAL OF MATHEMATICS, A NON-PROFIT CORPORATION}

The Supporting Institutions listed above contribute to the cost of publication of this Journal, but they are not owners or publishers and have no responsibility for its content or policies.

Reprinted 1966 in the United States of America 


\section{Pacific Journal of Mathematics}

\section{Vol. 11, No. 4}

A. V. Balakrishnan, Prediction theory for Markoff processes . . . . . . . . . . 1171

Dallas O. Banks, Upper bounds for the eigenvalues of some vibrating systems . . . . 1183

A. Białynicki-Birula, On the field of rational functions of algebraic groups ...... 1205

Thomas Andrew Brown, Simple paths on convex polyhedra .............. 1211

L. Carlitz, Some congruences for the Bell polynomials . . . . . . . . . . . . 1215

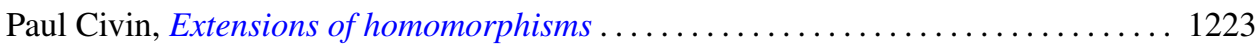

Paul Joseph Cohen and Milton Lees, Asymptotic decay of solutions of differential

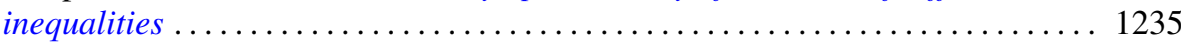

István Fáry, Self-intersection of a sphere on a complex quadric . . . . . . . . . . 1251

Walter Feit and John Griggs Thompson, Groups which have a faithful representation

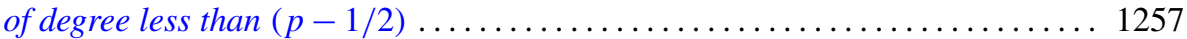

William James Firey, Mean cross-section measures of harmonic means of convex

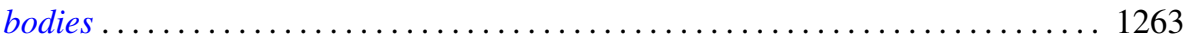

Avner Friedman, The wave equation for differential forms . . . . . . . . . . 1267

Bernard Russel Gelbaum and Jesus Gil De Lamadrid, Bases of tensor products of

Banach spaces ................................... 1281

Ronald Kay Getoor, Infinitely divisible probabilities on the hyperbolic plane . . . . 1287

Basil Gordon, Sequences in groups with distinct partial products . . . . . . . . . . . . 1309

Magnus R. Hestenes, Relative self-adjoint operators in Hilbert space . . . . . . . . . 1315

Fu Cheng Hsiang, On a theorem of Fejér ......................... 1359

John McCormick Irwin and Elbert A. Walker, On N-high subgroups of Abelian

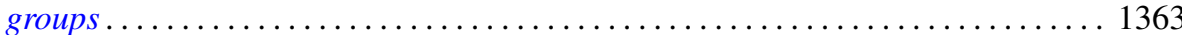

John McCormick Irwin, High subgroups of Abelian torsion groups . . . . . . . . . 1375

R. E. Johnson, Quotient rings of rings with zero singular ideal . . . . . . . . . . . 1385

David G. Kendall and John Leonard Mott, The asymptotic distribution of the time-to-escape for comets strongly bound to the solar system ...

Kurt Kreith, The spectrum of singular self-adjoint elliptic operators ....

Lionello Lombardi, The semicontinuity of the most general integral of the calculus of variations in non-parametric form ................................

Albert W. Marshall and Ingram Olkin, Game theoretic proof that Chebyshev inequalities are sharp

Wallace Smith Martindale, III, Primitive algebras with involution . . William H. Mills, Decomposition of holomorphs ..............

James Donald Monk, On the representation theory for cylindric algebras . . . . . . 1447

Shu-Teh Chen Moy, A note on generalizations of Shannon-McMillan theorem . . . . 1459

Donald Earl Myers, An imbedding space for Schwartz distributions . .

John R. Myhill, Category methods in recursion theory .........

Paul Adrian Nickel, On extremal properties for annular radial and circular slit mappings of bordered Riemann surfaces

Edward Scott O'Keefe, Primal clusters of two-element algebras . .

Nelson Onuchic, Applications of the topological method of Wazewski to certain

problems of asymptotic behavior in ordinary differential equations ...

Peter Perkins, A theorem on regular matrices................

Clinton M. Petty, Centroid surfaces .... 